\title{
Should we include microorganisms in scores to predict outcome in candidates for cardiac surgery during the acute phase of endocarditis?
}

\author{
Pierre Tattevin ${ }^{1}$, Pierre Fillâtre ${ }^{2}$, Serge Tchamgoué $^{3}$, Mathieu Lesouhaitier $^{1}$, Nicolas Nesseler ${ }^{4}$, \\ Jean-Marc Tadié ${ }^{1}$
}

${ }^{1}$ Infectious Diseases and Intensive Care Unit, Pontchaillou University Hospital, Rennes, France; ${ }^{2}$ Intensive Care Unit, Yves Le Foll Hospital, Saint Brieuc, France; ${ }^{3}$ Internal Medicine and Infectious Diseases, Robert Boulin Hospital, Libourne, France; ${ }^{4}$ Cardiovascular Thoracic Surgery Intensive Care Unit, Pontchaillou University Hospital, Rennes, France

Correspondence to: Pierre Tattevin. Professor, Infectious Diseases and Intensive Care Unit, Pontchaillou University Hospital, Service des Maladies Infectieuses et de Réanimation Médicale, CHU Pontchaillou, 2 rue Henri Le Guilloux, 35033 Rennes, France. Email: pierre.tattevin@chu-rennes.fr. Comment on: Williams JB, Shah AA, Zhang S, et al. Impact of microbiological organism type on surgically managed endocarditis. Ann Thorac Surg 2019. [Epub ahead of print].

Submitted Aug 27, 2019. Accepted for publication Sep 23, 2019.

doi: $10.21037 /$ jtd.2019.09.69

View this article at: http://dx.doi.org/10.21037/jtd.2019.09.69

Infective endocarditis (IE) is a complex disease, at the crossroad of a large number of medical specialties, including cardiology, infectious diseases, internal medicine, neurology, intensive care, microbiology, and radiology $(1,2)$. In addition, cardiac surgeons have been increasingly involved, as $60-70 \%$ of patients with IE present theoretical indication(s) for cardiac surgery (3). The selection of patients in whom cardiac surgery must be performed during the acute phase of IE is among the most difficult medical decisions in contemporary medicine, as both the benefits and the risks are high. Indeed, the strategy that will be finally selected, in agreement with other colleagues, the patient, and/or his relatives, may be a major determinant of whether the patient will die, or survive, with or without impairment of their quality of life.

To assist these tricky medical decisions, most clinicians use two complementary tools: (I) international guidelines from Europe (1), and/or America (3); (II) scores that have been developed from large cohorts, to predict outcome in patients candidates for cardiac surgery (4-23). The guidelines performed a complex process of weighing the pros, and the cons, for cardiac surgery, in a broad range of clinical situations commonly encountered in patients with IE. As could be expected, major indications for cardiac surgery are very similar in Europe and America guidelines, because the authors based their recommendations on the same tools, i.e., a comprehensive review of literature data, complemented by their opinions, as experts in the field. In brief, major indications for cardiac surgery are categorized in three groups in these guidelines: (I) heart failure, (II) uncontrolled infection, and (III) prevention of embolism $(1,2)$. However, the selection of patients who will benefit from cardiac surgery is much more complex than merely screening whether they present, or not, one of these indications: as for any major decision in medicine, the benefits of the intervention must be weighed against the risk(s). The specific situation of cardiac surgery during the acute phase of IE is even more complex, as the timing of cardiac surgery also plays an important role in the risk/benefit balance. As an example, guidelines state that early surgery should be considered in IE patients with large vegetation(s), for the prevention of embolism $(1,2)$. But they also outline literature data clearly demonstrating that the risk of embolism dramatically decreases following the start of appropriate antibiotics in patients with IE. As a consequence, the benefit of cardiac surgery for the prevention of embolism sharply decreases once an active anti-infective treatment is initiated, while the risk associated with cardiac surgery will remain grossly similar during the first days. Hence, a careful consideration of the risk/ benefit balance for this specific indication advocates for cardiac surgery to reduce embolism risk only if performed 
very early after appropriate anti-infective treatment has been started $(1,2)$. As stated by the European guidelines, 'the decision to operate early to prevent embolism is always difficult'. Then, they insist that, for this indication, 'surgery must be performed very early, during the first few days following initiation of antimicrobial treatment' (1). The situation is quite different when cardiac surgery is considered in patients with IE because of heart failure, or because of uncontrolled infection, as the situation will often get worse, with time, in patients who are not operated. In patients with life-threatening conditions (e.g., sepsis, or shock), early cardiac surgery is often a condition sine qua non for survival. For others, the decision may be postponed for a few days, when the indication, whether it is 'heart failure', or 'uncontrolled infection', is considered as 'borderline'. A proportion of them will finally be operated, as the potential benefit of cardiac surgery becomes more obvious, because for such indications, a few days of appropriate anti-infective treatment will not dramatically change the indication.

Only one randomized trial has been published, to better inform the decision to perform cardiac surgery during the acute phase of IE. However, patients enrolled in this unique trial were much younger than contemporary cohorts of patients with IE, they had less comorbidities, and inclusion criteria included a combination of two potential indications for cardiac surgery, i.e., severe valvular regurgitation, and vegetation $>10 \mathrm{~mm}$ (24). In this situation, early cardiac surgery has proven beneficial, but this only applies to a limited subgroup of patients, in whom the decision to operate is quite straightforward. A large number of observational studies, retrospective or prospective, aimed at better defining benefit/risk ratio of cardiac surgery during the acute phase of IE, but they present all the same major limitation: among patients with theoretical indications for cardiac surgery, those who are finally operated are not the same as those who are denied cardiac surgery $(3,25)$. Even high-level statistical methods, such as propensity-matched analysis cannot eliminate these potential biases.

All patients diagnosed with endocarditis should be screened to detect whether they present any indication for cardiac surgery. This screening is mandatory at diagnosis, and should be repeated in case of complications (e.g., heart failure, persistent bacteremia, progression of regurgitation or development of perivalvular abscess on echocardiography). The guidelines are particularly helpful for this process, although the decision to perform cardiac surgery in a patient with endocarditis cannot be based solely on guidelines: indeed, the second step in the decision- making process is the difficult evaluation of the risk/benefit ratio in an individual patient. At this stage, different scores that have been developed to predict outcome in patients candidates for cardiac surgery are used (4-23), although they all present limitations (Table 1): (I) those derived from cohorts of patients with heterogenous cardiac diseases may not be accurate for patients with IE (21); (II) early scores that were built by the time techniques for cardiac surgery, and intensive care were much different than current practices, probably overestimate major complications, including mortality $(4,5,8,14,17,21)$; (III) scores that have been validated in a specific cohort, with no validation in another group of patients, may not be generalizable.

To improve the performance of the scores currently available to predict outcome in IE patients candidates for cardiac surgery, Williams and colleagues performed a retrospective study of a large prospective cohort of patients who underwent cardiac surgery for IE during years 2011-2016 in North America, and were enrolled in the Society of Thoracic Surgeons Adult Cardiac Surgery Database (STS ACSD) (26). Their primary hypothesis was that the microorganisms responsible for IE have an impact on the prognosis of cardiac surgery, although to date no score took into account this parameter, readily available in $>90 \%$ of patients in most contemporary cohort studies. They developed univariable and risk-adjusted models with odds ratio (OR) for mortality for each organism type, taking 'streptococci' as the reference. To focus on adverse events most likely related to the surgical procedure, they used as primary endpoints: (I) operative mortality, using the STS ACSD standard definition: 'the greater of in-hospital, or 30-day mortality'; (II) STS major morbidity composite (stroke, deep sternal infection, prolonged ventilation, new onset renal failure, and/or reoperation); (III) hospital length of stay (26).

They could analyze 23,086 records for IE surgery, originating from 1,049 medical centers. Patients characteristics are in line with current epidemiology of IE patients who require cardiac surgery: median age was 56 years, $69 \%$ were males, with a large predominance of left-sided IE (93\%, n=21,388), mostly due to streptococci (32\%), staphylococci (30\%), and enterococci (15\%). They found that two microorganisms are independently associated with operative mortality, and 30-day major morbidity, in left-sided IE: (I) fungal IE, with adjusted OR of, respectively, 2.89 [95\% confidence interval (CI), 1.996-4.190], and 1.87 (95\% CI, 1.43-2.45), and (II) Staphylococcus aureus (S. aureus) IE, with OR of 1.41 (95\% 
Table 1 Risk scores for in-hospital mortality after surgery for IE

\begin{tabular}{|c|c|c|c|c|}
\hline Score & Derivation cohort size & $\begin{array}{l}\text { External } \\
\text { validation }\end{array}$ & Variables & Discrimination power (AUC) \\
\hline RISK-E score (18) & $\begin{array}{l}\mathrm{n}=424 \text { (multicentre, } \\
\text { left-sided IE only) }\end{array}$ & Yes & $\begin{array}{l}\text { Age, prosthetic valve IE, periannular } \\
\text { complications, Staphylococcus aureus or fungi } \\
\text { IE, acute renal failure, septic shock, cardiogenic } \\
\text { shock, thrombocytopenia }\end{array}$ & 0.82 (95\% Cl, 0.75-0.88) \\
\hline AEPEI score I (11) & $\mathrm{n}=361$ (multicentre) & Yes & $\begin{array}{l}\text { BMI }>27 \mathrm{~kg} / \mathrm{m}^{2}, \text { Egfr }<50 \mathrm{~mL} / \mathrm{mn}, \text { NYHA class IV, } \\
\text { sPAP }>55 \mathrm{mmHg} \text {, critical state }\end{array}$ & $0.780(95 \% \mathrm{Cl}, 0.734-0.822)$ \\
\hline AEPEI score II (11) & Idem & Idem & eGFR $<50 \mathrm{~mL} / \mathrm{mn}$, NYHA class IV, critical state & $0.774(95 \% \mathrm{Cl}, 0.727-0.816)$ \\
\hline PALSUSE score (16) & $\mathrm{n}=437$ (multicentre) & No & $\begin{array}{l}\text { Prosthetic valve IE, age } \geq 70 \text { years, large } \\
\text { intracardiac destruction, Staphylococcus spp IE, } \\
\text { urgent surgery, sex (female), EuroSCORE } \geq 10\end{array}$ & $0.84(95 \% \mathrm{Cl}, 0.79-0.88)$ \\
\hline De Feo score (22) & $\begin{array}{l}\mathrm{n}=440 \text { (single-centre, } \\
\text { prosthetic valve IE } \\
\text { excluded) }\end{array}$ & No & $\begin{array}{l}\text { Age, renal failure, NYHA class IV, ventilatory } \\
\text { support, positivity of latest preoperative blood } \\
\text { culture, perivalvular involvement }\end{array}$ & 0.88 \\
\hline
\end{tabular}

AUC, area under curve; IE, infective endocarditis; CI, confidence interval; BMI, body mass index; eGFR, estimated glomerular filtration rate; EuroScore, European system for cardiac operative risk evaluation; NYHA, New York heart association; sPAP, systolic pulmonary artery pressure; STS, Society of Thoracic Surgeons.

CI, 1.21-1.65) for operative mortality, and 1.42 (95\% CI, 1.30-1.56) for 30-day major comorbidity. Culture-negative IE were associated with operative mortality [OR, 1.35 (95\% CI, 1.10-1.66)], but not with major morbidity. They also found that operative mortality was higher for prosthetic valve left-sided IE, than for native valve left-sided IE (12\% vs. $8 \%, \mathrm{P}<0.0001)$. For right-sided IE, overall mortality was much lower (4\%), and ranking of mortality among main microorganisms tended to be similar (i.e., operative mortality of $6 \%, 4 \%$, and $2 \%$, for, respectively, fungal, $S$. aureus, and streptococci right-sided IE), but it didn't reach significance, so that the impact of microorganisms on postoperative adverse events could not be demonstrated.

The strength of this study includes the impressive number of observations available for analysis in the large STS ACSD database ( $n=23,086$ cases), with standardized definitions, regular training of data coordinators, quality control including annual on-site data audits, and adequate fulfilment of ethical and regulatory requirements. Rates of missing data appear to be very low (e.g., $0 \%$ for mortality), and causative organisms were reported for $94 \%$ of cases. These assets ensure that data are reliable, and results may be applied to most sites with similar epidemiology and practices. This study also has limitations. Firstly, by essence, only patients who were operated could be included in this database: hence, it provides no information on patients who had indications for cardiac surgery, but were not operated, whatever the reasons (major comorbidities, patients or relatives refusal, or death before surgery could be performed) (3). This is well illustrated by the rather low post-operative mortality ( $12 \%$ for prosthetic valve leftsided IE, $8 \%$ for native valve left-sided IE), which suggest that patients who were operated were indeed the most likely to survive, due to exclusion of patients with major comorbidities, or too severely ill due to IE, what is usually referred to as 'survival bias' (27). Secondly, information on microorganisms are sometimes not available by the times cardiac surgery must be decided, notably when 
microbiological diagnosis will be obtained through analysis of valve tissues (28). In that situation, not uncommon, data on microorganisms involved cannot be used as an early tool to predict post-operative complications. Thirdly, as the database was not primarily focused on microorganisms, important microbiological information are missing, including susceptibility (e.g., no information about methicillin-resistance for staphylococci), although this has a documented impact on patients outcome. However, the strengths of this study largely surpass the limitations, and the authors should be commended for this impressive amount of work, and the quality of original data presented.

Maybe the major criticism of this article would be the following: the authors merely confirmed, in the population of patients who are operated at the acute phase of IE, what has been known for decades in the overall population of patients with IE: S. aureus, and fungal IE (29), are associated with bad prognosis, whether they are operated or not. This also applies for the comparison of outcome for prosthetic valve IE versus native valve IE, or for left-sided IE versus right-sided IE. For the latter, even in the pre-antibiotic era, it has been documented that mortality was lower in right-sided IE ( $75 \%$, with a median survival of 27 days after diagnosis), than in left-sided IE [100\% mortality, median survival 12 days, reviewed in (30)]. Hence, although the data provided by Wiliams et al. are indeed original, they only confirm in a sub-group of patients previous findings in the overall population of patients with IE. The extrapolation of these findings in routine practice may not be dramatic: thanks to Williams et al. study, we now have robust data to support the higher risk of severe postoperative complications in patients with $S$. aureus, or fungal IE, who are candidates for cardiac surgery. However, these patients also have higher risk of bad outcome if they are not operated. Hence, the risk-benefit ratio may not be impacted by microbiological documentation, as microorganisms at higher risk of post-operative complications are also at risk of worse outcome in the absence of surgery.

In conclusions, despite the limitations detailed above, this study provides original data in the field, and suggests that additional variables may be incorporated in this complex decision-making process. Time will tell whether microorganisms will be integrated in future scores to predict post-operative complications in candidates for cardiac surgery during the acute phase of IE, and whether they will improve the performance of these scores, to assist clinicians in this difficult decision-making process

\section{Acknowledgments}

None.

\section{Footnote}

Conflicts of Interest: The authors have no conflicts of interest to declare.

Ethical Statement: The authors are accountable for all aspects of the work in ensuring that questions related to the accuracy or integrity of any part of the work are appropriately investigated and resolved.

\section{References}

1. Habib G, Lancellotti P, Antunes MJ, et al. 2015 ESC Guidelines for the management of infective endocarditis: the task force for the management of infective endocarditis of the European Society of Cardiology (ESC). Endorsed by: European Association for Cardio-Thoracic Surgery (EACTS), the European Association of Nuclear Medicine (EANM). Eur Heart J 2015;36:3075-128.

2. Baddour LM, Wilson WR, Bayer AS, et al. Infective endocarditis in adults: diagnosis, antimicrobial therapy, and management of complications: a scientific statement for healthcare professionals from the American Heart Association. Circulation 2015;132:1435-86.

3. Iung B, Doco-Lecompte T, Chocron S, et al. Cardiac surgery during the acute phase of infective endocarditis: discrepancies between European Society of Cardiology guidelines and practices. Eur Heart J 2016;37:840-8.

4. Alexiou C, Langley SM, Stafford H, et al. Surgical treatment of infective mitral valve endocarditis: predictors of early and late outcome. J Heart Valve Dis 2000;9:327-34.

5. Gabbieri D, Dohmen PM, Linneweber J, et al. Early outcome after surgery for active native and prosthetic aortic valve endocarditis. J Heart Valve Dis 2008;17:50824; discussion 525 .

6. Gaca JG, Sheng S, Daneshmand MA, et al. Outcomes for endocarditis surgery in North America: a simplified risk scoring system. J Thorac Cardiovasc Surg 2011;141:98106.e1-2.

7. Lalani T, Chu VH, Park LP, et al. In-hospital and 1 -year mortality in patients undergoing early surgery for prosthetic valve endocarditis. JAMA Intern Med 2013;173:1495-504. 
8. Langley SM, Alexiou C, Stafford HM, et al. Aortic valve replacement for endocarditis: determinants of early and late outcome. J Heart Valve Dis 2000;9:697-704.

9. Manne MB, Shrestha NK, Lytle BW, et al. Outcomes after surgical treatment of native and prosthetic valve infective endocarditis. Ann Thorac Surg 2012;93:489-93.

10. Pang PY, Sin YK, Lim CH, et al. Surgical management of infective endocarditis: an analysis of early and late outcomes. Eur J Cardiothorac Surg 2015;47:826-32.

11. Gatti G, Perrotti A, Obadia JF, et al. Simple scoring system to predict in-hospital mortality after surgery for infective endocarditis. J Am Heart Assoc 2017. doi: 10.1161/ JAHA.116.004806.

12. Chu VH, Park LP, Athan E, et al. Association between surgical indications, operative risk, and clinical outcome in infective endocarditis: a prospective study from the International Collaboration on Endocarditis. Circulation 2015;131:131-40.

13. Fernández-Hidalgo N, Ferreria-González I, Marsal JR, et al. A pragmatic approach for mortality prediction after surgery in infective endocarditis: optimizing and refining EuroSCORE. Clin Microbiol Infect 2018;24:1102.e71102.e15.

14. Hill EE, Herregods MC, Vanderschueren S, et al. Outcome of patients requiring valve surgery during active infective endocarditis. Ann Thorac Surg 2008;85:1564-9.

15. Madeira S, Rodrigues R, Tralhão A, et al. Assessment of perioperative mortality risk in patients with infective endocarditis undergoing cardiac surgery: performance of the EuroSCORE I and II logistic models. Interact Cardiovasc Thorac Surg 2016;22:141-8.

16. Martínez-Sellés M, Muñoz P, Arnáiz A, et al. Valve surgery in active infective endocarditis: a simple score to predict in-hospital prognosis. Int J Cardiol 2014;175:133-7.

17. Mestres CA, Castro MA, Bernabeu E, et al. Preoperative risk stratification in infective endocarditis. Does the EuroSCORE model work? Preliminary results. Eur J Cardiothorac Surg 2007;32:281-5.

18. Olmos C, Vilacosta I, Habib G, et al. Risk score for cardiac surgery in active left-sided infective endocarditis. Heart 2017;103:1435-42.

19. Patrat-Delon S, Rouxel A, Gacouin A, et al. EuroSCORE II underestimates mortality after cardiac surgery for infective endocarditis. Eur J Cardiothorac Surg 2016;49:944-51.

20. Rasmussen RV, Bruun LE, Lund J, et al. The impact of cardiac surgery in native valve infective endocarditis: can euroSCORE guide patient selection? Int J Cardiol 2011;149:304-9.

21. Roques F, Nashef SA, Michel P, et al. Risk factors and outcome in European cardiac surgery: analysis of the EuroSCORE multinational database of 19030 patients. Eur J Cardiothorac Surg 1999;15:816-22; discussion 822-3.

22. De Feo M, Cotrufo M, Carozza A, et al. The need for a specific risk prediction system in native valve infective endocarditis surgery. ScientificWorldJournal 2012;2012:307571.

23. Gatti G, Benussi B, Gripshi F, et al. A risk factor analysis for in-hospital mortality after surgery for infective endocarditis and a proposal of a new predictive scoring system. Infection 2017;45:413-23.

24. Kang DH, Kim YJ, Kim SH, et al. Early surgery versus conventional treatment for infective endocarditis. N Engl J Med 2012;366:2466-73.

25. Bannay A, Hoen B, Duval X, et al. The impact of valve surgery on short- and long-term mortality in left-sided infective endocarditis: do differences in methodological approaches explain previous conflicting results? Eur Heart J 2011;32:2003-15.

26. Williams JB, Shah AA, Zhang S, et al. Impact of microbiological organism type on surgically managed endocarditis. Ann Thorac Surg 2019. [Epub ahead of print].

27. Tattevin P, Uhel F, Fily F. Observational studies of salvage treatment for persistent bacteremia: beware of survivor treatment selection bias. Clin Infect Dis 2009;49:1960; author reply 1961.

28. Tattevin P, Watt G, Revest M, et al. Update on blood culture-negative endocarditis. Med Mal Infect 2015;45:1-8.

29. Tattevin P, Revest M, Lefort A, et al. Fungal endocarditis: current challenges. Int J Antimicrob Agents 2014;44:290-4.

30. Scheld WM. Therapy of streptococcal endocarditis: correlation of animal model and clinical studies. J Antimicrob Chemother 1987;20 Suppl A:71-85.

Cite this article as: Tattevin $\mathrm{P}$, Fillâtre $\mathrm{P}$, Tchamgoué $\mathrm{S}$, Lesouhaitier M, Nesseler N, Tadié JM. Should we include microorganisms in scores to predict outcome in candidates for cardiac surgery during the acute phase of endocarditis? J Thorac Dis 2019;11(10):E158-E162. doi: 10.21037/jtd.2019.09.69 